\title{
Evaluation of Local Filter Approaches for Diffusion Tensor-Based Fiber Tracking
}

\author{
D. Merhof ${ }^{1}$, M. Buchfelder ${ }^{2}$, C. Nimsky ${ }^{3}$ \\ ${ }^{1}$ Visual Computing, University Konstanz, Konstanz \\ 2 Department of Neurosurgery, University of Erlangen-Nuremberg, Erlangen \\ ${ }^{3}$ Department of Neurosurgery, University of Marburg, Marburg \\ dorit.merhof@uni-konstanz.de
}

\begin{abstract}
Diffusion tensor imaging (DTI) allows investigating white matter structures in vivo which is of great interest for various applications in neuroanatomy and neurosurgery. In order to reconstruct white matter tracts from DTI data, fiber tracking approaches are used which are commonly based on streamline propagation techniques. In order to provide more reliable tracking results, local regularization filters were presented previously, which can be applied during the fiber tracking process and move forward as the fibers are constructed. In this work, Gaussian and least-squares local filters are evaluated and compared against higher order integration schemes. The results provide a more differentiated view on the prospects and limitations of local regularization filters.
\end{abstract}

\section{Introduction}

Diffusion tensor imaging (DTI) is a magnetic resonance acquisition technique which provides information about the location of white matter tracts within the human brain in vivo. DTI measures the diffusion of water, which is anisotropic in tissue with a high degree of directional organization such as the white matter of the human brain. In this way, information about the location of neuronal fibers is provided which is of great interest for various applications in neuroanatomy and neurosurgery.

The reconstruction of white matter tracts from DTI data is commonly solved by tracking algorithms $[1,2]$ which use streamline techniques known from flow visualization. Respective algorithms propagate lines through the tensor field, based on the local diffusion characteristics which reflect the course of the underlying white matter tracts. The resulting fiber bundles approximate the location of neuronal fibers and are therefore a powerful tool for white matter reconstruction.

In recent years, local regularization techniques have been developed [3] with the aim to provide more reliable tracking results by preserving directional consistency and at the same time cross noisy regions and gaps in the data.

In this work, local filter approaches are evaluated and compared against higher order integration schemes, which also show the desirable feature of increased stability and tracking accuracy. 


\section{Material and Methods}

\subsection{Image Data}

The DTI datasets used in this work were acquired on a Siemens MR Magnetom Sonata Maestro Class 1.5 Tesla scanner using a field of view of $240 \mathrm{~mm}$ resulting in a voxel size of $1.875 \times 1.875 \times 1.9 \mathrm{~mm}^{3}$. For each of the six diffusion weighted datasets (gradient directions $( \pm 1,1,0),( \pm 1,0,1)$ and $(0,1, \pm 1))$ and the reference dataset, sixty slices with no intersection gap and an acquisition matrix of $128 \times 128$ pixels were measured.

\subsection{Fiber Tracking}

Fiber tracking algorithms based on streamline propagation have several steps in common such as seed point selection, fiber propagation and termination strategies [4]. Starting from predefined seed points, streamline integration is used to propagate the fiber until a termination criterion is reached. For this purpose, a threshold based on fractional anisotropy (FA) [1,2] is commonly applied to stop fiber propagation if the anisotropy of diffusion decreases.

In this work, fiber tracts were computed using first, second and fourth order Runge-Kutta integration and trilinear tensor interpolation. A constant tracking step size of $0.5 \mathrm{~mm}$ was employed. FA was used as termination threshold for fiber propagation, and a length threshold of a minimum of 100 tracking steps was used in order to exclude short fibers from the tracking result.

\subsection{Local Regularization Filters}

Local regularization filters [3] are applied during the fiber tracking process and move forward as the fibers are constructed. In order to put more weight on the tensors in the direction of the tracked fiber, a moving filter window of ellipsoidal shape centered around the current end point of the fiber is employed (Fig. 1). The size of the filter window is adjusted depending on the reliability of the local tensor data.

During fiber tracking, a local regularization approach is applied to the tensors in the filter window in order to approximate the local tensor. The resulting regularized tensor is used for tract propagation.

In order to weight the tensors within the filter window, a Gaussian weighting function $G\left(\boldsymbol{s}-\boldsymbol{p} ; \boldsymbol{D}_{p}\right)$ is introduced [3], which assigns a weight to a point position

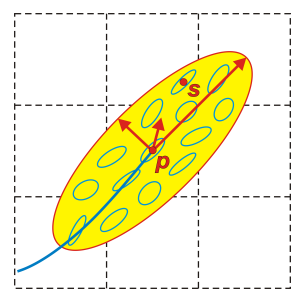

Fig. 1. Local filter window domain (yellow). The filtered tensor at the center $\boldsymbol{p}$ is computed from samples at different locations $\boldsymbol{s}$ within the filter window. 
$\boldsymbol{s}$ in the vicinity of the center point $\boldsymbol{p}$. The weighting function has the effect that tensors located close to the center of the filter window have a higher impact on the regularization result than tensors at the border of the filter window. The shape of the Gaussian filter window is adapted from the tensor ellipsoid associated with the local tensor $\boldsymbol{D}_{p}$ at the center point $\boldsymbol{p}$.

In order to approximate the regularized tensor $\overline{\boldsymbol{D}}_{p}$ at position $\boldsymbol{p}$ using the Gaussian filter window, the weighted average of the tensors within the filter volume is computed.

Moving least-squares filter The local Gaussian filtering scheme is extended by a least-squares approximation within the filter window [3]. Least-squares approximation aims at minimizing an error functional $E$ which measures the quality of a polynomial fit of the data

$$
E(\boldsymbol{p})=\frac{1}{V} \int_{V} G\left(\boldsymbol{s}-\boldsymbol{p} ; \boldsymbol{D}_{p}\right) \cdot[\boldsymbol{F}(\boldsymbol{s}-\boldsymbol{p})-\boldsymbol{D}(\boldsymbol{s})]^{2} d \boldsymbol{s}^{3}
$$

In order to solve this equation, a low degree polynomial $\boldsymbol{F}$ has to be found which best approximates the data $\boldsymbol{D}(\boldsymbol{s})$ at different locations $\boldsymbol{s}$ within the filter window centered around $\boldsymbol{p}$. The quality of fit in a least-squares sense is assessed by the functional $E$, which must be minimized. The impact of a point location on the functional $E$ is controlled by the filter function $G\left(\boldsymbol{s}-\boldsymbol{p} ; \boldsymbol{D}_{p}\right)$.

For fiber tracking, the original, noisy tensor $\boldsymbol{D}_{p}$ is approximated with the polynomial fit $\boldsymbol{F}$ at position $\boldsymbol{p}$.

\section{Results}

Fiber tracking was performed both with and without local filters. In Figure 2, the result of fiber tracking without local filtering (left), with a Gaussian local
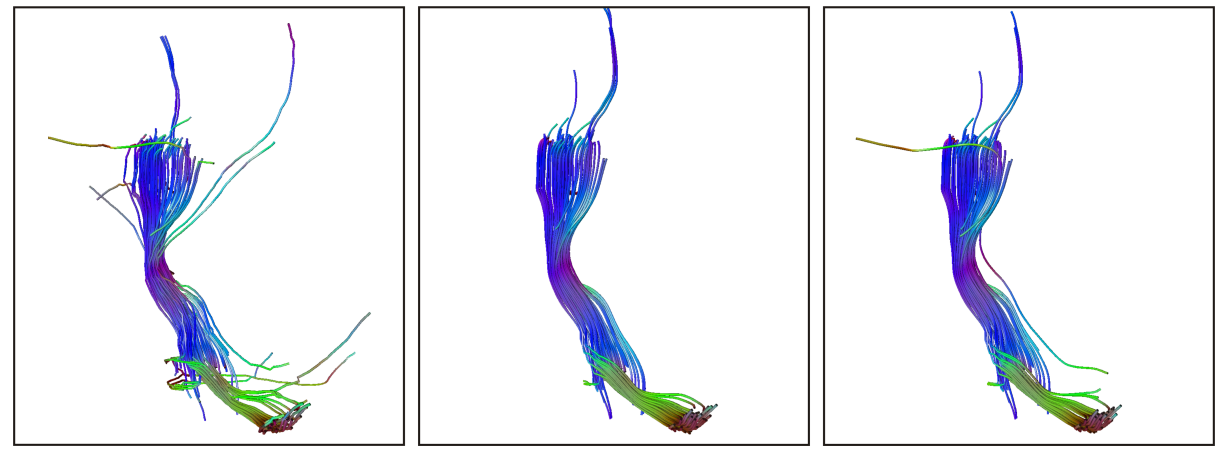

Fig. 2. Pyramidal tract in a healthy volunteer reconstructed with streamline integration (RK-1) without filtering (left), with a Gaussian local regularization filter (middle), and with a moving least-squares filter (right). 
Table 1. Accuracy and computing time for oriented filters. Time: Computing time for different integration schemes without filtering (left) and with a Gaussian local regularization filter and a moving least-squares filter (right) for a tracking of the whole brain. Divergence: Average divergence between the original fiber and the reverse fiber after 50 integration steps.

\begin{tabular}{lll}
\hline & Time & Divergence \\
\hline RK-1 none & $4 \mathrm{sec}$ & $8.61 \mathrm{~mm}$ \\
RK-2 none & $11 \mathrm{sec}$ & $1.46 \mathrm{~mm}$ \\
RK-4 none & $18 \mathrm{sec}$ & $1.27 \mathrm{~mm}$ \\
\hline
\end{tabular}

\begin{tabular}{lll}
\hline & Time & Divergence \\
\hline RK-1 none & $4 \mathrm{sec}$ & $8.61 \mathrm{~mm}$ \\
RK-1 Gauss & $242 \mathrm{sec}$ & $5.91 \mathrm{~mm}$ \\
RK-1 MLS & $464 \mathrm{sec}$ & $5.88 \mathrm{~mm}$ \\
\hline
\end{tabular}

regularization filter (middle) and with a moving least-squares filter (right) is shown. In each case, streamline propagation using Euler integration (RK-1) was employed. For both local filtering approaches, the moving filter windows were scaled with a factor $\sigma=50$ and tensor samples within the filter window were taken at a distance of $1 \mathrm{~mm}$ for each dimension in 3D space. In the case of the least-squares filter, a polynomial of degree 2 was applied for the approximation.

In order to assess the quality of local filtering, both techniques were compared to fiber-tracking results without filtering. For this purpose, streamlines were traced until a termination criterion was reached, and the final sampling point of the fiber was used as the starting point for a reverse fiber, running in the opposite direction. Depending on the quality of the tracking approach applied, the reverse fiber closely follows the original fiber for a certain number of steps, before it diverges from the course of the original fiber. As a consequence, the distance between corresponding sampling points of the original fiber and the reverse fiber indicates the quality of the respective tracking approach. In order
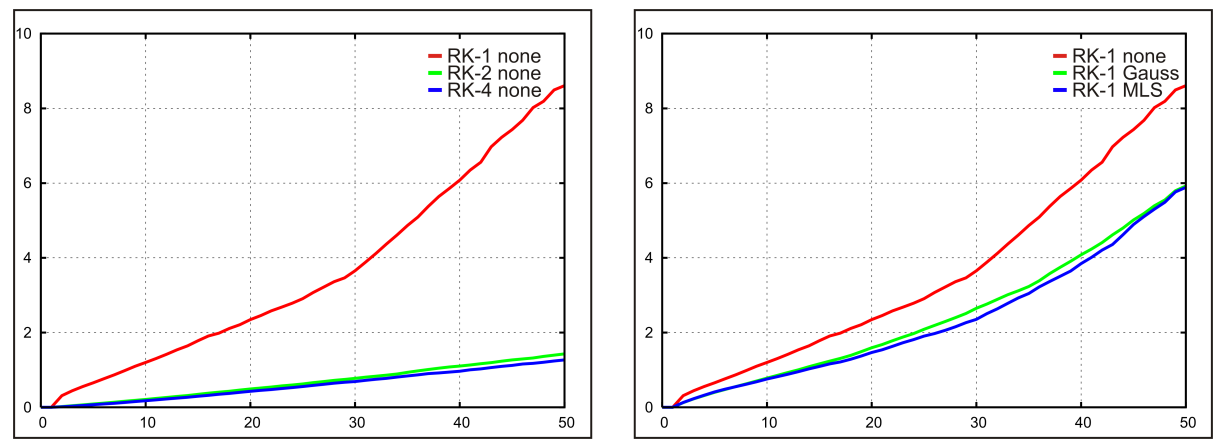

Fig. 3. Divergence between original fiber and reverse fiber for 50 integration steps, averaged for all fibers in a tracking of the whole brain. Left: Comparison of different integration schemes (RK-1, RK-2 and RK-4). Right: Comparison of RK-1 integration without local filter (RK-1 none), with local Gaussian filter (RK-1 Gauss) and with moving least-squares filter (RK-1 MLS). 
to obtain significant results, approximately 300.000 streamlines within the whole brain and associated reverse fibers were computed and the results were averaged. The diagram in Figure 3 (right) shows the averaged divergence between original and reverse fibers for streamline propagation without filtering, with a Gaussian local regularization filter and with a moving least-squares filter. In Table 1, the divergence after 50 integration steps as well as the computing times on a PC equipped with an Intel Pentium 4, $3.4 \mathrm{GHz}$, and $2 \mathrm{~GB}$ RAM are provided.

\section{Discussion}

From the diagram in Figure 3 (right), it can be concluded that the Gaussian local regularization filter provides similar accuracy to that of the moving least-squares filter, i.e. the computational effort added by the least-squares approximation has only a very slight impact on the accuracy. Taking into account the computing time in Table 1 (right), which is much higher in the case of least-squares approximation, it can be concluded that the Gaussian local regularization filter is much more suitable for practical application.

In principle, the local filters improve the tracking accuracy of the respective integration scheme, as shown in Figure 3 (right) for streamline tracking based on first order Runge-Kutta integration. However, for a higher-order integration scheme, such as second or fourth order Runge-Kutta in Figure 3 (left), even greater accuracy is obtained compared to the lower-order integration in combination with local filters. For this reason, it is more feasible to apply higher order integration instead of a RK-1 integration scheme combined with local filters.

\section{Conclusion}

In this work, local regularization filters were evaluated and compared against higher order integration schemes. For both the Gaussian local filter and the least-squares local filter, significantly increased computing times were encountered compared to higher order integration schemes. In terms of tracking accuracy, higher order integration schemes outperform RK-1 integration with local regularization filters. In summary, it can be concluded that higher order integration is preferable to local regularization filters.

\section{References}

1. Basser PJ, Pajevic S, Pierpaoli C, et al. In vivo fiber tractography using DT-MRI data. Magn Reson Imaging. 2000;44(4):625-632.

2. Mori S, Crain BJ, Chacko VP, et al. Three-dimensional tracking of axonal projections in the brain by magnetic resonance imaging. Ann Neurol. 2001;45(2):265-269.

3. Zhukov L, Barr A. Oriented Tensor Reconstruction: Tracing Neural Pathwaysfrom Diffusion Tensor MRI. In: Proc IEEE Vis; 2002. p. 387-394.

4. Mori S, van Zijl PCM. Fiber tracking: principles and strategies: a technical review. NMR Biomed. 2002;15(7-8):468-480. 